Z. Phys. Chem. 216 (2002) 589-603

(C) by Oldenbourg Wissenschaftsverlag, München

\title{
Femtosecond Fluorescence Anisotropy Kinetics as a Signature of Ultrafast Electronic Energy Transfer in Bichromophoric Molecules
}

\author{
By Vlastimil Fidler ${ }^{1, *}$, Peter Kapusta ${ }^{1}$, Miloš Nepraš ${ }^{2}$, Jörg Schroeder ${ }^{3}$, \\ Igor V. Rubtsov ${ }^{4, * *}$ and Keitaro Yoshihara ${ }^{4}$ \\ ${ }^{1}$ Faculty of Nuclear Sciences and Physical Engineering, Czech Technical University in \\ Prague, V Holešovičkách 2, 18000 Prague 8, Czech Republic \\ 2 Department of Organic Technology, University of Pardubice, Nám. Čs. legií 565, \\ 53210 Pardubice, Czech Republic \\ 3 Department of Spectroscopy and Photochemical Kinetics, Max-Planck-Institute of \\ Biophysical Chemistry, D-37070 Göttingen, Germany \\ ${ }^{4}$ School of Materials Science, Japan Advanced Institute of Science and Technology, \\ 1-1 Asahidai, Tatsunokuchi, Ishikawa, 923-12 Japan
}

Dedicated to Prof. Dr. Dr. h.c. mult. Jürgen Troe on the occasion of his 60th birthday

(Received August 18, 2000; revised version accepted April 12, 2001)

\section{Intramolecular Electronic Energy Transfer / Bichromophore / Vibronic Coupling / IVR}

Ultrafast time resolved fluorescence anisotropy decay measurements were performed to gain insight into the energy gap dependence of donor-acceptor inter-chromophoric coupling within one supra-molecule. Three new compounds, each consisting of two semirigidly linked and strongly coupled chromophores, were designed and synthesized for this study. Their general structure is donor-spacer-acceptor, where "donor" is phenylamino, pyrenylamino, or benzanthronylamino moiety, and acceptor is aminobenzanthrone. While being similar structurally, the compounds differ significantly in the excitation energy difference of the two chromophores in a supra-molecule. Experimental data show an ultrafast initial fluorescence emission anisotropy decrease (within less then $1 \mathrm{ps}$ ) when the excited state energies of the interacting chromophores are close to each other or equal. No such fast fluorescence anisotropy dynamics is observed for a compound with a large energy gap.

* Corresponding author. E-mail: Vlastimil_Fidler@brown.edu

** Present address: Chemistry Department, University of Pennsylvania, Philadelphia, PA, USA 


\section{Introduction}

Electronic interactions between ground and excited states of separate molecules or distinct parts of a molecule or a supramolecular structure are at the basis of a variety of phenomena in reaction dynamics and photophysics which play a key role in many light-induced processes in nature and technical applications. Among the important photophysical processes, one of the most widely studied is electronic energy transfer (EET) in its different forms that occurs on characteristic time scales covering more than ten orders of magnitude from about $10^{-14}$ to $10^{-2}$ seconds which reflects the range of systems and physical conditions over which EET is observed. From a molecular engineering point of view, intramolecular EET in suitably designed compounds incorporating two or more photophysically active subunits appears particularly attractive, since one has structural control over EET sensitive parameters such as, e.g., distance and relative orientation of transition dipoles. In this sense, rigidly coupled molecular groups in particular appear suitable to systematically investigate the details of energy transfer dynamics, which in the case of intermolecular EET involving bimolecular systems in solution usually is obscured by translational and rotational diffusion. Both rigid and flexible coupled bichromophoric systems have been investigated in solution and under isolated molecule conditions in supersonic jet expansions, and the results of such studies were discussed extensively in a recent excellent review [1].

As for intermolecular EET in solution, also in intramolecular EET both well-known basic types of EET play a role: long-range transfer mediated by Coulomb interaction, i.e. the so-called Förster mechanism [2-5], and shortrange transfer as a consequence of exchange interaction as proposed by Dexter [6]. While the details of the Coulombic Förster mechanism seem to be well understood (see Ref. [1] and references cited therein), exchange interaction induced intramolecular EET - including "classical" short range, super exchange [7] and through bond mechanisms [8,9] - is the subject of numerous both theoretical and experimental investigations (see Ref. [1] for a review), in part because of its relevance for, e.g., primary steps in photosynthesis $[10,11]$ or the design of molecular electronic devices [12,13].

The ideal bichromophoric compound consists of two distinct chromophore units separated by a spacer that spatially separates the subunits in a welldefined way and at the same time is electronically decoupled from both of them. Thus, the absorption spectrum of the bichromophore should just be the superposition of the corresponding spectra of the two chromophores. To avoid "trivial" dipole-dipole transfer, the relevant electronic transitions should be weak and the spectral overlap should be essentially zero. Upon electronic excitation intramolecular coupling should become non-negligible, such that exciplex formation [14], intramolecular charge $[15,16]$ or electronic energy transfer $[1,16]$ are induced. The specific design of the system will determine which of these processes will contribute or dominate. The signature of intramolecu- 
lar EET is a strong or complete quenching of donor fluorescence accompanied by a corresponding appearance of acceptor emission and a dual fluorescence decay consisting of a fast component due to the quenched donor emission and a contribution from the EET-induced acceptor excited state fluorescence. Ideally, to unambiguously demonstrate efficient intramolecular EET, one would have to observe an ultrafast decay of donor fluorescence and a matching rise of acceptor emission, with a time constant directly related to the rate of EET.

Recently, we reported on a new class of semi-rigidly linked bichromophoric compounds including a structure consisting of 1-aminopyrene and 3-aminobenzanthrone chromophoric subunits connected by a chlorotriazinyl spacer that fulfils these basic requirements as judged by the corresponding absorption spectra [17] to some extent, though the triazinyl group has a non-negligible influence on the photophysics of both donor and acceptor [18]. Regarding their flexibility, the attribute "semi-rigid" places these compounds between highly flexible aliphatic chain linked molecules and those held together by fully rigid bridges such as, e.g., polynorbornyls [16]. We studied in detail the photophysics of two groups of fluorophores: N-substituted 1-aminopyrenes [19] and $\mathrm{N}$-substituted 3-aminobenzanthrones. Also, the basic spectroscopic properties of a new bichromophoric compound connecting two fluorophores of these types via triazinyl spacers were investigated in order to construct a model compound exhibiting predominantly intramolecular EET and negligible electron transfer. More than 25 model compounds (including specifically designed and synthesized molecules) were investigated for this purpose. A rather detailed understanding of the photophysics of both 3-aminobenzanthrones (the acceptor moiety in the bichromophore) and 1-aminopyrenes (the corresponding donor moiety) was achieved, and spectroscopic steady state as well as sub-nanosecond time resolved studies of the aminopyrene-chlorotriazineaminobenzanthrone bichromophore indicated extremely fast EET within this supramolecule. We also presented first direct evidence from femtosecond time resolved experiments for ultrafast intramolecular EET taking place in this compound on a sub-picosecond time scale [18]. Nonetheless, the precise mechanism responsible for such rapid EET and the nature of interactions between the fluorophores acting as electronic energy donor and acceptor across the optically inactive spacer have to be clarified in more detail. In this paper we will present new unambiguous evidence for ultrafast EET based on comparison with a symmetrical bichromophore and steady state and time resolved fluorescence anisotropy studies.

\section{Experimental}

\section{Syntheses}

3-nitrobenzanthrone was prepared by nitration of benzanthrone $(7 \mathrm{H}-\mathrm{Benz}$ [de] anthracene-7-one, CAS no. 82-05-3) with $87 \% \mathrm{HNO}_{3}$, in nitrobenzene at $40-50{ }^{\circ} \mathrm{C}$; m.p. $242-245^{\circ} \mathrm{C}$. 
3-aminobenzanthrone ( $\mathrm{ABa}$ hereafter) was prepared by reduction of 3-nitrobenzanthrone with $\mathrm{Na}_{2} \mathrm{~S} \cdot 9 \mathrm{H}_{2} \mathrm{O}$ in water suspension at $90-100{ }^{\circ} \mathrm{C}$; m.p. $238-239^{\circ} \mathrm{C}$.

2-(3-benzanthronylamino)-4,6-dichloro-1,3,5-triazine ( $\mathrm{ABa}-\mathrm{TC}_{2}$ hereafter) was prepared from $\mathrm{ABa}$ and cyanuric chloride (2,4,6-trichloro-1,3,5-triazine, CAS no. 108-77-0) in acetone with $\mathrm{NaHCO}_{3}$ and a small amount of water by stirring at $5-10^{\circ} \mathrm{C}$ for $90 \mathrm{~min}$. The product was filtered, washed with ethanol and water and recrystallized from toluene; m.p. $>340{ }^{\circ} \mathrm{C}$.

2-(1-pyrenylamino)-4,6-dichloro-1,3,5-triazine (APy- $\mathrm{TC}_{2}$ hereafter) was prepared by the same procedure as $\mathrm{ABa}-\mathrm{TC}_{2}$, but using 1-aminopyrene (CAS no. 1606-67-3) instead of $\mathrm{ABa}$ as a reactant; m.p. $236-237^{\circ} \mathrm{C}$.

2-(3-benzanthronylamino)-4-(phenylamino)-6-chloro-1,3,5-triazine (AnTC-ABa hereafter) was prepared from $\mathrm{ABa}^{-} \mathrm{TC}_{2}$ and aniline (CAS no. 62-53-3) in 1,3-dimethyl-2-imidazolidinone (DMI, CAS no. 80-73-9) solvent. The reaction mixture was stirred at room temperature for several hours and then poured into water. The precipitated product was separated and washed with ethanol and water; m.p. $\geq 330^{\circ} \mathrm{C}$.

2,4-di(3-benzanthronylamino)-6-chloro-1,3,5-triazine (ABa-TC-ABa hereafter) was prepared from $\mathrm{ABa}-\mathrm{TC}_{2}$ and $\mathrm{ABa}$ by stirring and refluxing in $\mathrm{DMI}$ for 48 hours (until the red color of $\mathrm{ABa}$ disappears). The reaction mixture was then poured into water. The precipitated product was washed with acetone; m.p. $>340{ }^{\circ} \mathrm{C}$.

2-(3-benzanthronylamino)-4-(1 - pyrenylamino)-6-chloro-1,3,5-triazine (APy-TC-ABa) was prepared by the same procedure as ABa-TC-ABa from $\mathrm{APy}-\mathrm{TC}_{2}$ and $\mathrm{ABa}$; m.p. $>340^{\circ} \mathrm{C}$. Further details of the synthesis and characterization of the bichromophoric dyes used in this study [20-22] will be published elsewhere .

\section{Sample preparation}

Samples for steady-state fluorescence measurements were prepared by preparative TL chromatography on Silufol UV 254 silica plate. For absorption and fluorescence up-conversion measurements (where higher sample concentrations are necessary), several times re-crystallized compounds were used. UV spectroscopic grade dimethyl sulfoxide (DMSO) was used as a solvent. For steady-state fluorescence anisotropy measurements the samples were dissolved in purified 2-methyltetrahydrofurane, placed in a $1 \mathrm{~cm}$ diameter cylindrical quartz cuvette and cooled down to $77.15 \mathrm{~K}$ in Oxford Instruments Optistat DN cryostat.

\section{Steady-state spectroscopic measurements}

Absorption spectra were measured with a Shimadzu UV 3100 UV/VIS/NIR spectrometer. Typical concentrations were of the order of $10^{-5} \mathrm{M}$, which 
yielded optical densities of about 0.3 at the main absorption maximum in a $1 \mathrm{~cm}$ cuvette. Corrected steady-state fluorescence excitation and emission spectra were measured using an FS/FL900 photon counting spectrofluorometer from Edinburgh Instruments. For fluorescence measurements, the sample absorbance at any excitation wavelength was kept well below 0.1 . The monochromator bandwidths were $1 \mathrm{~nm}$ FWHM for fluorescence excitation/emission spectral measurements, and $2 \mathrm{~nm}$ FWHM for fluorescence anisotropy measurements, respectively. Fluorescence quantum yields were determined by integrating the area under the emission spectrum and comparing it with that of quinine sulphate standard solution $\left(q_{\mathrm{F}}=0.54\right.$ in $\left.0.1 \mathrm{~N} \mathrm{H}_{2} \mathrm{SO}_{4}\right)$. Saturating the solution with $\mathrm{N}_{2}$ or Ar did not change spectra and measured quantum yields, therefore the reported data correspond to aerated solutions.

\section{Time-Resolved Fluorescence Measurements}

Sub-nanosecond fluorescence decay kinetics were measured using the timecorrelated single photon counting (TCSPC) method, either by an Edinburgh Instruments FS/FL900 spectrofluorometer set-up [19,23] or by a home-built TCSPC set-up described previously [24]. Fluorescence kinetics were measured under magic-angle conditions; spectral bandwidth (FWHM) used at FS/FL900 was $10 \mathrm{~nm}$, at the system described in [24] dye-laser excitation and relevant cut-off filters were used. Sub-picosecond fluorescence decay data was acquired by fluorescence up-conversion. As the general method has been described in the literature (see, e.g., $[25,26]$ ), we only give details of the specific experimental set-up used for the measurements described here. A Ti-sapphire oscillator-amplifier system (Clark-MXR, CPA-1000) pumped by a CW multiline Ar-ion laser (Spectra Physics Beamlok) operated at $100 \mathrm{MHz}$ was used as a source of short laser pulses. After external compression by a prism pair, pulses as short as $30 \mathrm{fs}$ were obtained at wavelengths around $800 \mathrm{~nm}$. The second harmonic $(\mathrm{SH})$ was generated in a 0.3 or $0.5 \mathrm{~mm}$ BBO crystal, and recompressed by another prism pair down to 35 fs. The SH pulses (pulse energy $<1 \mathrm{~nJ}$, bandwidth $7 \mathrm{~nm}$ FWHM) were focused into a thin sample cell by a spherical mirror $(f=5 \mathrm{~cm})$ to excite the sample. Fluorescence was collected by a 90 degrees parabolic aluminum mirror and focused into the up-conversion crystal (BBO, Type I, $0.5 \mathrm{~mm}$ thickness) by a second parabolic mirror. The pulses at the fundamental frequency, passed through an optical delay line, were used as gate pulses. The up-converted light at the sum frequency was spatially and spectrally filtered, passed through a monochromator (15 nm FWHM bandwidth), and detected by a photomultiplier (R585S from Hamamatsu Photonics) working in the photon-counting regime. The fundamental wavelength of the oscillator could be tuned from 740 to $870 \mathrm{~nm}$. The time-resolution of the set-up reached $70 \mathrm{fs}$ as judged by the FWHM of the instrument response function (IRF) at about $800-840 \mathrm{~nm}$ with a $0.3 \mathrm{~mm}$ up-conversion crystal. For shorter wavelengths the IRF increased significantly. For the shortest wave- 


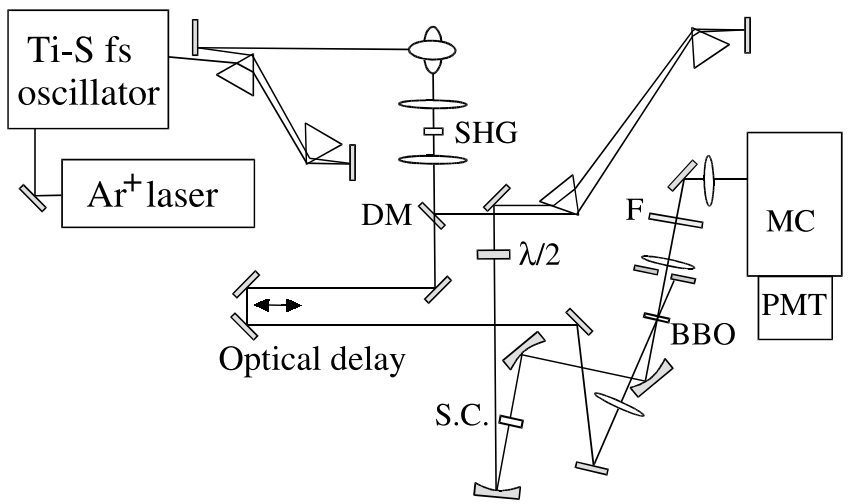

Fig. 1. Experimental set-up used for femtosecond fluorescence up-conversion measurements.

length used in this study (i.e. $750 \mathrm{~nm}$ ) the IRF was about $190 \mathrm{fs}$ (using a $0.5 \mathrm{~mm}$ up-conversion crystal). The polarization of the gate pulses was fixed, while a half-wave plate controlled that of the pump pulses. More than ten scans with alternate polarization of the pump pulses were run and averaged to get anisotropy data. For anisotropy measurements, the steric angle of the fluorescence collection was limited to about 0.02 steradian. The samples were circulated in a $0.5 \mathrm{~mm}$ flow cell (marked as SC in Fig. 1) and were carefully tested for degradation. No signs of any photodegradation were found under the low-energy excitation used.

\section{Results and discussion}

Fig. 2 shows the steady state spectra of three bichromophoric compounds we will consider, 2-(3-benzanthronylamino)-4-(phenylamino)-6-chloro1,3,5-triazine (An-TC-ABa), 2-(3-benzanthronylamino)-4-(1-pyrenylamino)6-chloro-1,3,5-triazine (APy-TC-ABa), and 2,4-di(3-benzanthronylamino)-6chloro-1,3,5-triazine (ABa-TC-ABa), where the energy gap between the two chromophores decreases in the order from An-TC-ABa, APy-TC-ABa to $\mathrm{ABa}-\mathrm{TC}-\mathrm{ABa}$. The absorption spectra are normalized with respect to the maximum of the absorption band corresponding to the acceptor (TC-ABa) part of the spectrum in the 400 to $450 \mathrm{~nm}$ region. The absorption spectrum of $\mathrm{ABa}-\mathrm{TC}_{2}$ (i.e. the parent compound with a second chlorine atom on the triazine instead of aniline) is added to the top panel to illustrate that the anilino-group has a negligible contribution above $310 \mathrm{~nm}$. Absorption spectra in the middle part of Fig. 2. illustrate that the APy-TC-ABa bichromophore absorption is approximately a superposition of the corresponding model subunits spectra represented here by those of An-TC-ABa and APy- 

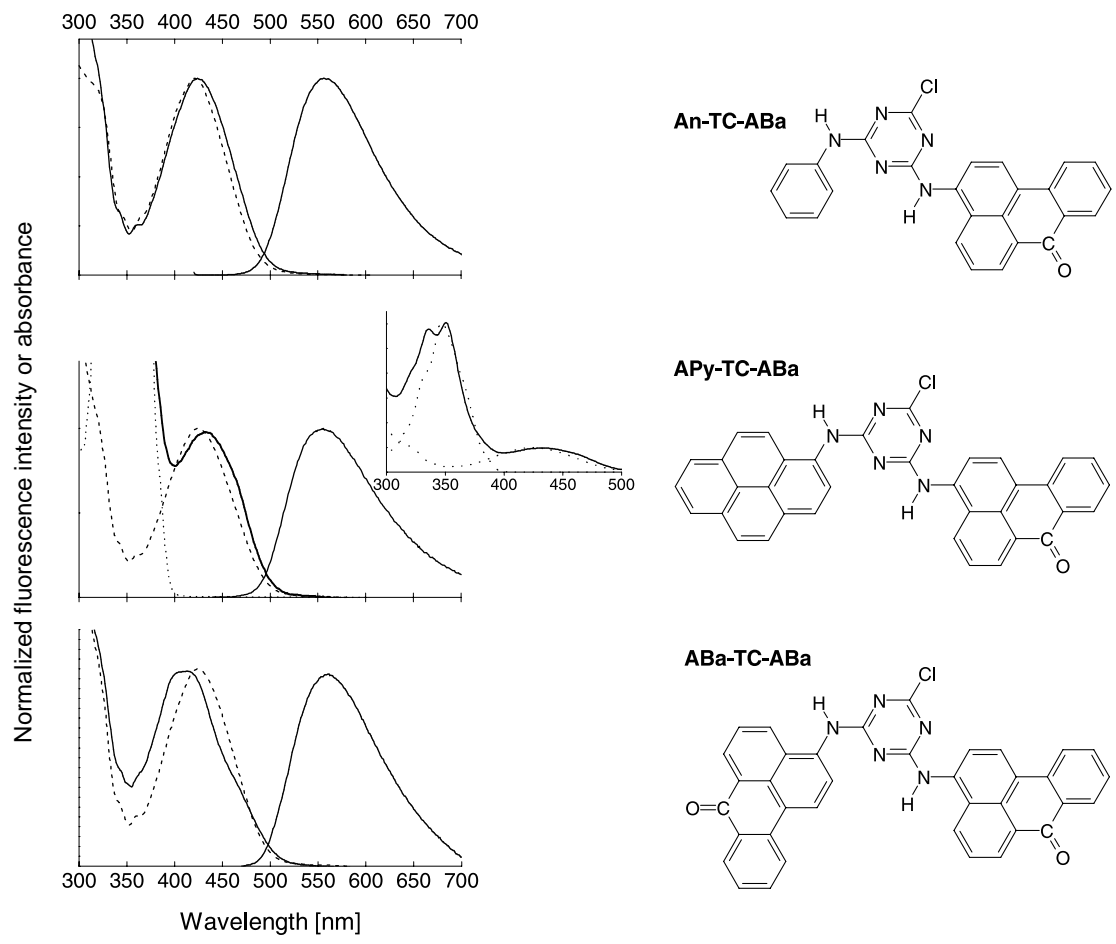

Fig. 2. Chemical structures and normalised steady-state absorption and fluorescence spectra (solid lines) of the compounds studied. The absorption spectrum of $\mathrm{ABa}^{-\mathrm{TC}_{2}}$ (dashed line) in the top panel illustrates that the anilino-group has a negligible contribution above $310 \mathrm{~nm}$. Likewise, the absorption spectra of APy-TC-An (model donor, dotted line) and An-TC-ABa (model acceptor, dashed line) in the middle panel demonstrate the spectral superposition. The inset with the rescaled absorption spectra of APy-TC-ABa and the model compounds illustrates the relative absorbance of the two parts involved in energy transfer. The bottom panel includes the absorbtion spectrum of An-TC-ABa (model "onehalf" of ABa-TC-ABa, dashed line) again to show the spectral changes of the lowest wavelength band.

TC-An. It should be stressed here that these anilino derivatives (An-TC-ABa and APy-TC-An), though being bichromophoric compounds themselves, could be considered as good model compounds of virtual chromophoric subunits of APy-TC-ABa since their anilino groups chemically mimic the situation in the bichromophore. The APy-TC-ABa absorbance at $350 \mathrm{~nm}$ is almost exclusively due to the corresponding donor subunit APy-TC. For the third, symmetrical compound $\mathrm{ABa}-\mathrm{TC}-\mathrm{ABa}$, the bottom panel of Fig. 2 shows the shape and slight broadening of the lowest wavelength absorption band. Comparison with the An-TC-ABa absorbtion band (included as a dashed line) suggests presence of splitting due to resonance interaction across the spacer unit. 
Fluorescence emission spectra shown in Fig. 2 for all three compounds represent the only fluorescence emission that can be detected for any excitation wavelength from 250 to $500 \mathrm{~nm}$. Spectral positions and emission band shapes correspond to those of the TC-ABa part [18].

The signature of the electronic states responsible for acceptor type fluorescence emission in the $550 \mathrm{~nm}$ band after excitation at different wavelengths is clearly visible in the steady state excitation anisotropy spectra of the three compounds presented in Fig. 3. For An-TC-ABa, the high degree of practically constant anisotropy in the 360 to $400 \mathrm{~nm}$ excitation range clearly indicates that the initially excited state is identical to the emitting state, while the sharp drop in anisotropy at $360 \mathrm{~nm}$ and its spectral dependence for shorter wavelengths

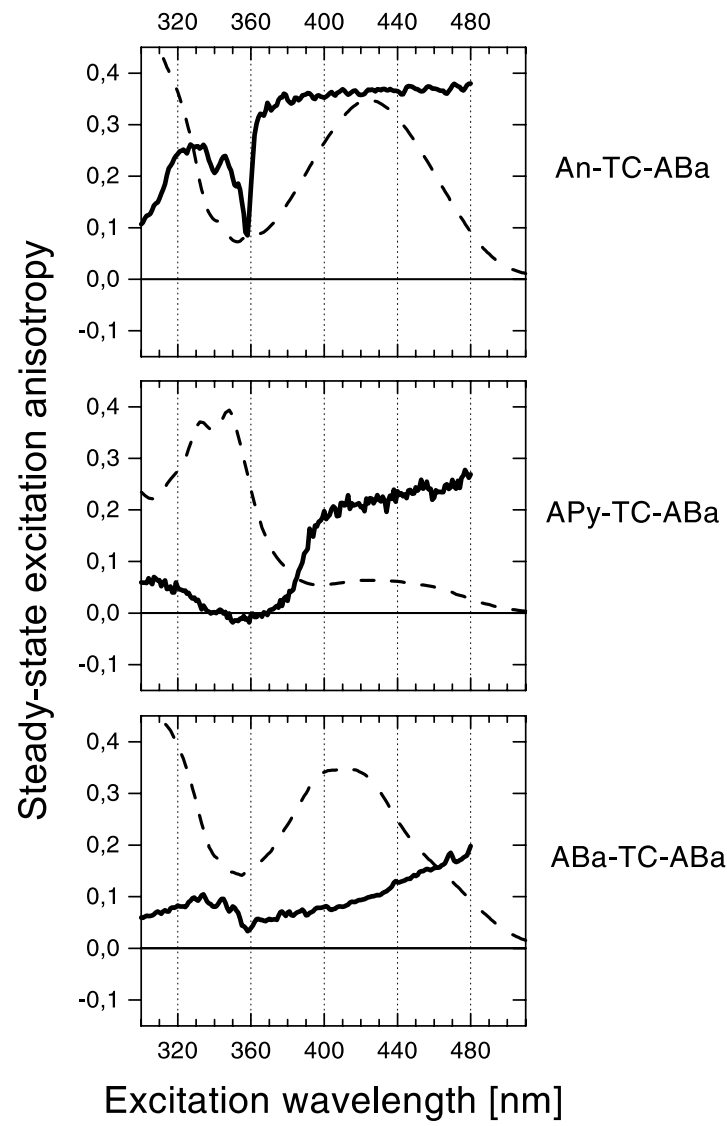

Fig. 3. Steady-state fluorescence excitation anisotropy spectra (acceptor emission is detected at $520 \mathrm{~nm}$, solid lines) of An-TC-ABa, APy-TC-ABa, and ABa-TC-ABa, from top to bottom, respectively. Corresponding absorption spectra are indicated by dashed lines. 
signal excitation of electronic states other than the emitting state, in accordance with the absorption spectrum. For APy-TC-ABa the situation is different: the degree of anisotropy in 400 to $480 \mathrm{~nm}$ region is smaller and decreases more pronouncedly towards shorter wavelengths. This is a clear indication that the directly excited emitting state of the acceptor subunit is not as "pure" as in AnTC-Aba, i.e. it is affected by electronic interaction with the donor part. The drop of excitation anisotropy already at $370-380 \mathrm{~nm}$ signals transition to donor subunit excitation as expected from looking at the absorption spectra depicted on Fig. 2. In the symmetric compound ABa-TC-ABa, the indications of significant electronic coupling across the spacer unit are manifested by the overall low degree of excitation anisotropy, which is in accordance with the ABa-TC$\mathrm{ABa}$ absorption spectrum as shown on Fig. 2.

The anisotropy spectra of An-TC-ABa and ABa-TC-ABa compounds clearly show a fairly localized minimum at $360 \mathrm{~nm}$, which coincides with the weak $S_{3} \leftarrow S_{0}$ transition in the TC-ABa acceptor subunit [18]. At this stage it is not clear, whether or to what extent this state is involved in the EET process in the APy-TC-ABa bichromophoric compound. In the 310-390 nm region, the APy-TC-ABa fluorescence excitation anisotropy is mostly governed by the APy-TC (donor part). Common feature is the monotonous decrease (towards shorter wavelengths) of anisotropy in the long wavelength region that may be associated with increasing vibronic coupling to other electronic states as the excess excitation energy above the origin in the initially excited local acceptor state increases. This effect, of course, is more pronounced in compounds that show indications of stronger donor-acceptor electronic coupling already at vanishing excess energy.

Fig. 4 shows acceptor subunit fluorescence decay curves measured under magic angle conditions after excitation at $403 \mathrm{~nm}$. Though this selection of excitation wavelength was influenced by experimental reasons as well, according to Fig. 2 and Fig. 3, at $403 \mathrm{~nm}$ one still achieves practically exclusive local excitation of the acceptor subunit TC-ABa.

For An-TC-ABa the decay is purely monoexponential with a lifetime of $(6.7 \pm 0.1) \mathrm{ns}$ in DMSO solution, confirming the notion of a pure locally excited acceptor state. The fluorescence decay of APy-TC-ABa is much faster, i.e. the fluorescence is significantly quenched from a fluorescence quantum yield of 0.28 in An-TC-ABa to a value of 0.05 . Also the decay is no longer monoexponential, but can be fitted by a double exponential with lifetimes of $(60 \pm 5)$ ps and $(360 \pm 20)$ ps. Evidently, the emitting state of the acceptor part is significantly coupled to the APy-TC unit. The consequences of coupling are even more evident in the symmetric compound, where the fluorescence quantum yield is further reduced to a value of 0.01 . Also, the fluorescence decay is now clearly double exponential with the longer lifetime of $(350 \pm 130)$ ps being comparable to the corresponding value of APy-TC-ABa emission within experimental accuracy (i.e. taking into account the large uncertainty of this component for a fit on the given time scale), and a fast compon- 


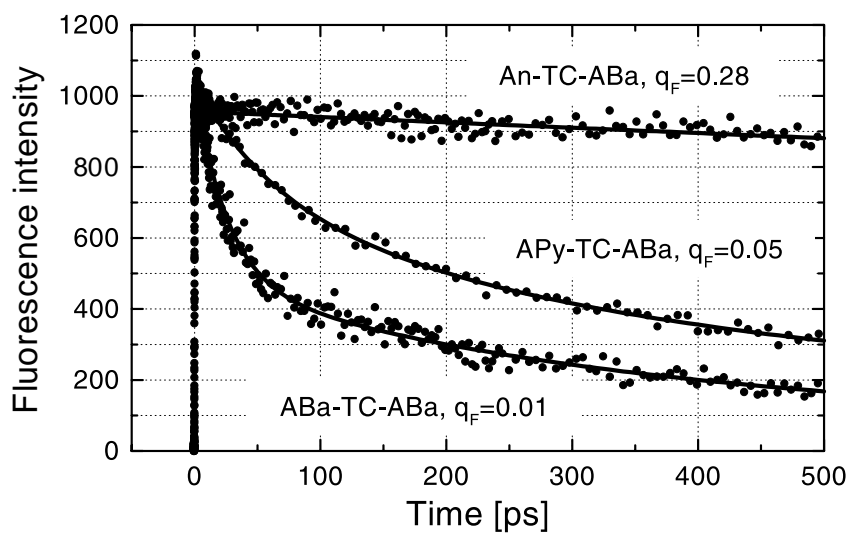

Fig. 4. Initial part of bichromophore fluorescence decay kinetics in DMSO solution at room temperature measured by fluorescence up-conversion. The emission was monitored at $520 \mathrm{~nm}$ following excitation at $403 \mathrm{~nm}$. The polarization plane of the excitation beam was set to magic angle with respect to the polarisation plane of the probe beam to avoid fluorescence depolarization effects during these measurements. The corresponding steady state fluorescence quantum yields are also indicated. The lines indicate the fitted decays using the lifetimes given in the text.

ent with a lifetime of $(26 \pm 2)$ ps, roughly half of the corresponding value for APy-TC-ABa.

Although it seems that another electronic state coupled to the locally excited acceptor state is responsible for fluorescence quenching, the observation of non-exponential decays is surprising, as a straightforward quenching process would just increase the first order decay rate constant. One can imagine different possibilities that could explain this observation. In the electronically coupled systems excitation at $403 \mathrm{~nm}$ might populate two coupled states: (1) that emit in the same wavelength region and have different lifetimes, or (2) one of which is dark and subsequently slowly repopulates the emitting state. In the latter case, one would expect an initial fast component due to faster depletion of the emitting state in favor of the dark state that would have to be slightly lower in energy. This internal conversion would be followed by immediate fast vibrational relaxation to thermal equilibrium in the dark state and subsequent thermally activated repopulation of the emitting state. Without additional information it is not possible to decide which description is closer to reality.

Fig. 5 illustrates the sub-picosecond acceptor emitting state population rise time in APy-TC-ABa for different excitation wavelengths. The fastest rise is observed for excitation at $403 \mathrm{~nm}$, i.e. direct absorption by the acceptor unit. The 10 to 90 percent rise time of 200 fs probably reflects a convolution of the IRF of the up-conversion set-up with intramolecular dynamics that also seem to dominate the subsequent $200 \mathrm{fs}$, before the signal decays due to loss of excited 


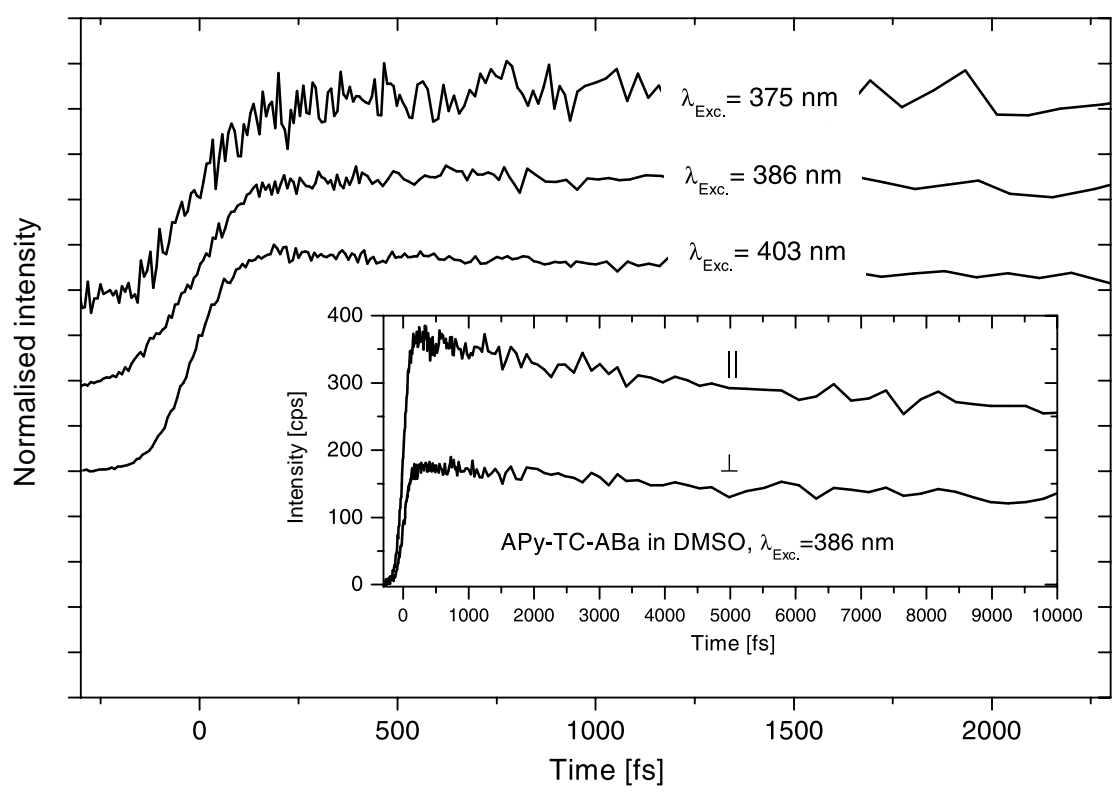

Fig. 5. Comparison of APy-TC-ABa femtosecond fluorescence kinetics detected at $525 \mathrm{~nm}$ for various excitation wavelengths. The curves were shifted vertically to facilitate comparison. Inset shows typical polarized fluorescence decays used for calculation of anisotropy kinetics.

state population. The key observation, however, is that the rise of the acceptor emission is almost as fast, if at $375 \mathrm{~nm}$ one almost exclusively excites the donor moiety. The 10 to 90 percent rise time in this case is as short as about $300 \mathrm{fs}$, a clear indication of ultrafast interchromophoric EET. Again, other intramolecular processes such as, e.g., intramolecular vibrational energy redistribution (IVR) may also contribute to the observed temporal profile. In this sense, the rate constant of populating the acceptor excited state obtained from the fluorescence rise, $k_{\mathrm{EET}} \approx 3 \cdot 10^{12} \mathrm{~s}^{-1}$, represents a lower limit to the true EET rate coefficient.

Another logical way to monitor EET kinetics would be donor fluorescence kinetics measurement. Such an approach was not applicable in our case, since residual fluorescence of the donor part was detectable not even on a short time scale. Fortunately enough, a key test to verify the occurrence of intramolecular EET in such a bichromophoric system consists of determining the time resolved fluorescence anisotropy by measuring emission time profiles with parallel and perpendicular polarization with respect to the exciting laser beam, as indicated in the inset of Fig. 5. As molecular rotational motion practically is frozen on the time scale of our ultrafast measurements, the time resolved fluorescence emission anisotropy provides a straightforward way to monitor 


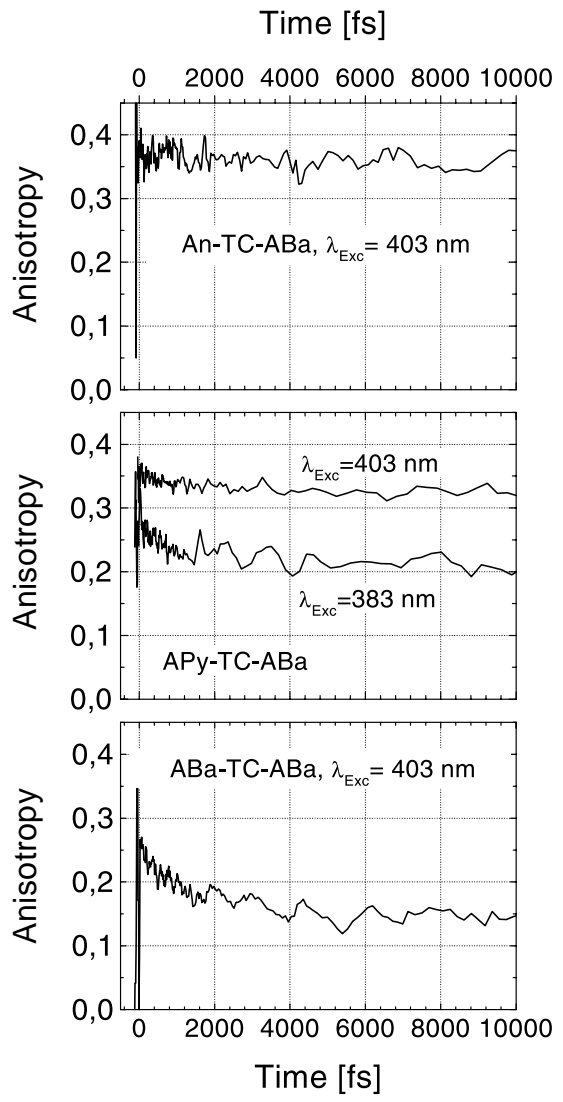

Fig. 6. Time evolution of fluorescence anisotropy of the -TC-ABa moiety in DMSO solvent at room temperature. The curves were calculated from $\|$ and $\perp$ polarized fluorescence decays measured at $520 \mathrm{~nm}$ by fluorescence up-conversion. The excitation wavelength is indicated at each curve.

the temporal evolution of emitting states involved. This is possible, because the relative orientation of transition moment vectors of the relevant electronic states remains fixed in the rigid bichromophore and the emission anisotropy value just depends on the relative orientation of initial absorption and final emission transition moments, and not on acceptor excited state population. The graph at the center of Fig. 6 illustrates the result of such measurements of the acceptor emission for APy-TC-ABa in DMSO solvent. Excitation at $403 \mathrm{~nm}$, i.e. of the acceptor moiety, of course leads to an instantaneous anisotropy value of 0.35 , close to the theoretical maximum expected when transition moments of absorbing and emitting states are collinear. Excitation at $383 \mathrm{~nm}$, however, initially populates the donor excited state mainly, whereas that of the acceptor is only populated subsequently by ultrafast EET. This leads to a much 
lower initial degree of polarization of the emission, reflected in an anisotropy value of only about 0.27 . This observation supports the conclusion that EET indeed is responsible for the fast acceptor emission rise. The interpretation of the initial acceptor fluorescence anisotropy rests on the assumption that depolarization within the donor moiety prior to energy transfer is negligible, as so far there seem to be no signs of corresponding dynamic effects. We will, however, pursue this subject in future studies of the donor moiety. The occurrence of extremely efficient EET in this bichromophore is in line with the observation of significant enhancement of EET in rigidly with respect to flexibly linked bichromophoric systems $[1,27]$. The order of magnitude of the transfer rate coefficient definitely points towards a through bond superexchange mechanism, but a detailed account of this will be given in connection with suitable model calculations. In any case, Förster type EET in no way could account for the ultrafast transfer process. An attempt to estimate $k_{\mathrm{EET}}$, Förster value was made, based on Förster quenching measurements with model donor and model acceptor (4,6-dimethoxy derivatives of APy-T.. and ABa-T..) mixture in ethyl acetate that lead to a critical transfer radius value of $2.7 \mathrm{~nm}$ and donor lifetime $\tau_{\mathrm{D}} \approx 4$ to $5 \mathrm{~ns}$, and further based on a distance between the two transition moments of 13 to $15 \AA$ estimated from APy-TC-ABa molecular structure. It yielded a maximum estimated value of $k_{\mathrm{EET} \text {, Förster }} \approx \leq 2 \cdot 10^{10} \mathrm{~s}^{-1}$, i.e. two orders of magnitude smaller than the observed lower limit of the rate coefficient.

It is also interesting to note that the anisotropy slightly decreases within a couple of picoseconds before it attains a quasi-constant value on this time scale. Following excitation at $403 \mathrm{~nm}$, the effect is small, corresponding to an anisotropy reduction of just ten percent from its initial value. We think that it probably is a signature of enhanced vibronic coupling of donor and acceptor moieties across the spacer immediately after excitation, i.e. before complete IVR has led to a microcanonical distribution of vibrational energy within the acceptor. If such a picture were realistic, one would expect that the phenomenon would be more pronounced at higher initial excitation energies. Indeed, at an excitation wavelength of $383 \mathrm{~nm}$ the effect is significantly stronger. The initial anisotropy decay is faster, and the anisotropy reduction after 10 ps amounts to about 25 percent of the initial maximum. This is consistent with the expectation that vibronic coupling is stronger and that IVR will be faster initially due to the larger amount of excess energy. Also, different vibrational modes could be populated in direct excitation and following EET from donor to acceptor.

In order to study the role of vibronic coupling between donor and acceptor across the spacer, we also investigated the time dependence of the acceptor fluorescence anisotropy for the other two compounds An-TC-ABa and ABaTC-ABa. After $403 \mathrm{~nm}$ excitation, in An-TC-ABa where the donor-acceptor energy gap is large, one expects little, if any, vibronic donor-acceptor coupling. Indeed, one observes a practically constant high anisotropy value of about 0.36 for this compound within the first $10 \mathrm{ps}$. In the symmetric compound elec- 
tronic energy levels are degenerate and, as discussed above, evidence for strong coupling is already evident in the absorption spectrum, the steady state fluorescence excitation anisotropy spectrum, and in the picosecond fluorescence decay. This also shows up in the low value of the initial anisotropy of 0.25 (bottom graph in Fig. 6), and its marked decrease to about 50 percent of the initial value within a couple of picoseconds. As the excess energy is roughly the same as for $403 \mathrm{~nm}$ acceptor excitation of the bichromophore APy-TC-ABa, the time constants of the decays are very close to each other, which seems reasonable as IVR has to occur on a similar time scale.

\section{Conclusions}

We have now convincing evidence based on steady state and femtosecond timeresolved spectroscopic data that in the bichromophoric compound APy-TC$\mathrm{ABa}$ ultrafast EET takes place from the initially excited aminopyrene moiety across the triazin spacer to the aminobenzanthrone moiety. We were able to directly determine a lower limit of the EET rate coefficient of $3 \cdot 10^{12} \mathrm{~s}^{-1}$ for this rigidly linked compound which indicates an efficient through bond EET mechanism. The comparison of time resolved fluorescence anisotropy traces of all three compounds suggests that EET occurs prior to IVR and most probably is enhanced by excitation of specifically coupled vibrational modes.

\section{Acknowledgement}

Financial support of the 202/98/0566 grant by the Czech State Grant Agency is gratefully acknowledged, together with our thanks to the Japan Society for the Promotion of Science and the Ministry of Education, Youth and Sports of the Czech Republic for their support of the Japan-Czech collaboration in molecular sciences (grant KONTAKT ME269).

\section{References}

1. S. Speiser, Chem. Rev. 96 (1996) 1953.

2. Th. Förster, Ann. Phys. (Leipzig) 2 (1948) 55.

3. Th. Förster, Z. Naturforsch. 4a (1949) 321.

4. Th. Förster, in Modern Quantum Chemistry, O. Sinanoglu ed., Academic Press, New York 1968, p. 93.

5. M. D. Galanin, Sov. Phys. JETP 1 (1955) 317.

6. D. L. Dexter, J. Chem. Phys. 21 (1953) 836.

7. E. M. Monberg and R. Kopelmann, Chem. Phys. Lett. 58 (1978) 492.

8. R. Hoffmann, Acc. Chem. Res. 4 (1971) 19.

9. J. Klafter and J. Jortner, Chem. Phys. Lett. 60 (1978) 5.

10. K. P. Ghiggino and T. A. Smith, Progr. React. Kin. 18 (1993) 375.

11. G. D. Scholes, I. R. Gould, R. J. Cogdell and G. R. Fleming, J. Phys. Chem. B 103 (1999) 2543. 
12. V. Balzani and F. Scandola, Supramolecular Photochemistry, Harwood Ellis, New York 1991.

13. I. V. Rubtsov, Y. Kobuke, H. Miyaji and K. Yoshihara, Chem. Phys. Lett. 308 (1999) 323.

14. J. Michl and V. Bonacic-Koutecky, Electronic Aspects of Organic Photochemistry, VCH, New York 1990.

15. M. D. Newton, Chem. Rev. 91 (1991) 767.

16. K. D. Jordan and M. N. Paddon-Row, Chem. Rev. 92 (1992) 395.

17. M. Nepraš, O. Machlický, M. Šeps, R. Hrdina, P. Kapusta and V. Fidler, Dyes and Pigments 35 (1997) 31.

18. V. Fidler, P. Kapusta， M. Nepraš，J. Schroeder，I. V. Rubtsov and K. Yoshihara, Coll. Czech. Chem. Commun. 63 (1998) 1460.

19. P. Kapusta, V. Fidler, M. Nepraš, P. Šoustek and M. Hof, in Fluorescence Microscopy and Fluorescence Probes, A. Kotyk ed., Espero Publishing, Prague 1999, p. 145.

20. N. Salem, M. Sc.-Thesis, University of Pardubice, 1992.

21. P. Markuzelová, M. Sc.-Thesis, University of Pardubice, 1996.

22. P. Šoustek, M. Sc.-Thesis, University of Pardubice, 1997.

23. P. Kapusta, Ph. D.-Thesis, Czech Technical University, 1998.

24. C. Gehrke, R. Mohrschladt, J. Schroeder, J. Troe and P. Vöhringer, Chem. Phys. 152 (1991) 45.

25. G. R. Fleming, Chemical Applications of Ultrafast Spectroscopy, OUP, New York 1986.

26. B. E. A. Saleh and M. C. Teich, Fundamentals of Photonics, Wiley, New York 1991.

27. S. Speiser and F. Schael, J. Molec. Liq. 86 (2000) 25. 